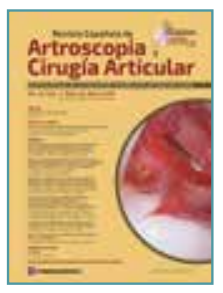

Vol. 25. Fasc. 1. Núm. 61. Marzo 2018

ISSN: 2386-3129 (impreso)

2443-9754 (online)

\title{
Revisión
}

\section{Revisión sistemática: ligamento anterolateral de la rodilla}

\author{
A. Gómez¹, D. García-Germán², A. Espejo Reina³, \\ E. López ${ }^{4}$, I. Tamimi ${ }^{1}$, A. Espejo Baena ${ }^{3}$
}

\author{
${ }^{1}$ Hospital Regional Universitario de Málaga \\ ${ }^{2}$ Hospital Universitario Puerta de Hierro Majadahonda. Madrid \\ ${ }^{3}$ Hospital Vithas Parque San Antonio. Málaga \\ ${ }^{4}$ Hospital Quirónsalud Málaga \\ Correspondencia: \\ Dr. Abel Gómez Cáceres \\ Correo electrónico: gomezcaceresabel@gmail.com
}

Recibido el 23 de noviembre de 2017

Aceptado el 14 de febrero de 2018

Disponible en Internet: marzo de 2018

\section{RESUMEN}

Introducción: numerosos estudios han sido realizados en los últimos años con el objetivo de mejorar el conocimiento del ligamento anterolateral (LAL) de la rodilla. Debido a esto, se cree conveniente realizar esta revisión bibliográfica para definir la incidencia, la anatomía, la biomecánica, la clínica, las pruebas de imagen y el tratamiento del mismo.

Material y métodos: se realiza una búsqueda en las bases de datos PubMed/Medline/Cochrane. Se incluyeron estudios descriptivos tanto clínicos como in vitro sobre las consideraciones anatómicas, biomecánicas, clínicas, estudios de imagen, manejo quirúrgico y resultados del LAL de la rodilla hasta abril de 2017. Se excluyen artículos no escritos en inglés, publicados en revistas no indexadas, no realizados en humanos o cadáveres humanos y aquellos no pertenecientes a la rodilla.

Resultados: el origen femoral es el que más discusión nos ofrece, insertándose en la tibia entre el tubérculo de Gerdy y la cabeza del peroné. Biomecánicamente, parece influir en la estabilidad rotacional, con escaso control de la estabilidad anteroposterior. Para su diagnóstico, disponemos del pivot shift en la exploración, fractura de Segond en la radiología simple, ecografía y resonancia magnética.

\begin{abstract}
Systematic review: anterolateral ligament of the knee

Introduction: numerous studies have been performed in the last years with the aim of improving the knowledge on the anterolateral ligament (ALL) of the knee. It is therefor deemed necessary to perform this literature revision in order to define its incidence, anatomy, biomechanics, clinical, image studies and treatment of the same one.
\end{abstract}

Material and methods: a PubMed/Medline/Cochrane data base search is performed. Descriptive studies, both clinical and in vitro, on anatomy, biomechanics, clinical presentation, imaging findings, surgical treatment and results of the ALL of the knee up to april 2017. Paper not written in English, published in non-indexed journals, not done on humans or human cadavers, or not referring to the knee were excluded.

Results: the femoral origin is less clear than the tibial insertion occurring between Gerdy's tubercle and the fibular head. Biomechanicaly ALL controls rotational stability with little effect over antero-posterior translation. As diagnostic tools, we have the pivot-shift at clinical exploration, Segond's fracture in plain X-rays, ultrasonography

FS $\odot 2018$ Fundación Española de Artroscopia. Publicado por Imaidea Interactiva en FONDOSCIENCE ${ }^{\circledR}$ (www.fondoscience.com) Este es un artículo Open Access bajo la licencia CC BY-NC-ND (www.creativecommons.org/licenses/by-nc-nd/4.0/). 
Para el tratamiento de esta estructura se utilizan plastias extraarticulares y reconstrucciones del LAL asociadas a la reconstrucción del ligamento cruzado anterior (LCA).

Discusión: la inestabilidad rotacional residual tras la reconstrucción del LCA empeora los resultados y las técnicas quirúrgicas han ido evolucionando para evitarla. Hoy en día, la existencia de estructuras anterolaterales es evidente, por lo que la reconstrucción de la misma asociada al LCA parece ser una técnica a tener en cuenta en pacientes con alto riesgo de pivot shift postoperatorio.

Palabras clave: Ligamento anterolateral. Pivot shift. Fractura de Segond. Tenodesis extraarticular.

\section{Introducción}

Desde la reciente publicación de Claes ${ }^{(1)}$ sobre el ligamento anterolateral (LAL), numerosos estudios han sido realizados con el objetivo de mejorar el conocimiento de esta estructura.

La primera mención a este ligamento fue realizada en 1879 por Paul Segond(2) al describir una "pearly, resistant, fibrous band" en la zona anterolateral de la rodilla causante de la fractura-avulsión de Segond. En décadas posteriores, múltiples nombres han sido utilizados para definir a esta estructura, "lateral capsular ligament", "midlateral capsular ligament", "midthird lateral capsular ligament" o "capsulo-osseous layer of the iliotibial $\operatorname{tract}^{\prime(3-6)}$. El término ligamento anterolateral fue utilizado por primera vez en 2007 por Vieira et al. ${ }^{(7)}$.

La inserción femoral de este ligamento es la que más discusión nos ofrece, con 3 localizaciones descritas(1,8-15): sobre el epicóndilo, proximal y posterior, y anterior y distal a este. La localización tibial ofrece menos discusión, situándola en la meseta tibial externa, entre en tubérculo de Gerdy y la cabeza del peroné.

Los estudios biomecánicos ponen de manifiesto el papel de esta estructura en el control de la estabilidad rotacional, existiendo debate sobre la posición donde se produce mejor función biomecánica por la misma. Parece ser que presenta mayor tensión en grados cercanos a la exten$\operatorname{sió}^{(1,8-10,13,16,17)}$, pero estudios recientes defienden su control a mayor flexión de rodilla ${ }^{(15,18-21)}$.

Para el diagnóstico de esta lesión se ha puesto de manifiesto ser el causante del pivot shift en and magnetic resonance imaging. Extraarticular lateral tenodesis and ALL reconstructions associated to anterior cruciate ligament (ACL) reconstructions are used.

Discussion: residual rotational instability worsens the results after ACL reconstruction, surgical techniques have evolved to avoid it. The existence of the anterolateral structures is clear now and its reconstruction should be considered in patients with high risk of postoperative pivot-shift.

Keywords: Anterolateral ligament. Pivot-shift. Segond's fracture. Extraarticular tenodesis.

la exploración, aunque este concepto es contro-

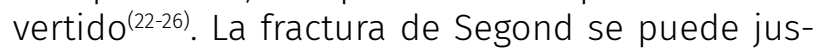
tificar como una avulsión de este ligamento en su inserción tibial ${ }^{(9,27,28)}$. La resonancia magnética $(\mathrm{RM})^{(8,28-30)}$ y la ecografía(31-33) están siendo utilizadas para caracterizar este ligamento.

Para su tratamiento se dispone de diversas técnicas de refuerzo extraarticular con diferentes tipos de injerto ${ }^{(34-37)}$, obteniendo buenos resultados tras un seguimiento de 24 meses $^{(38)}$.

Debido a la gran cantidad de estudios con respecto a este ligamento en los últimos años, se cree conveniente realizar esta revisión bibliográfica para definir la incidencia, la anatomía, la biomecánica, la clínica, las pruebas de imagen y el tratamiento del mismo.

\section{Metodología}

\section{Criterios de inclusión}

Se incluyeron estudios descriptivos tanto clínicos como in vitro sobre las consideraciones anatómicas, biomecánicas, clínicas, estudios de imagen, manejo quirúrgico y resultados del LAL de la rodilla.

\section{Estrategia de búsqueda}

Se realizó una búsqueda en las bases de datos PubMed/Medline/Cochrane con los parámetros "(anterolateral ligament OR ALL OR lateral capsular ligament) AND (injuries OR anatomy OR bio- 
mechanics OR instability OR pivot shift OR Segond fracture OR MRI OR ultrasound OR reconstructions)". Las consultas con expertos y la búsqueda manual de las referencias de los artículos fueron utilizadas para identificar estudios adicionales elegibles. También se incluyen los resúmenes de las conferencias organizadas por las siguientes sociedades: American Academy Of Orthopaedic Surgeons (AAOS), International Society of Arthroscopy, Knee Surgery and Orthopaedic Sports Medicine (ISAKOS) y European Society of Sports Traumatology, Knee Surgery and Arthroscopy (ESSKA). Se seleccionaron los estudios descriptivos hasta el mes de abril de 2017. También se incluyeron estudios publicados antes de su impresión.

\section{Selección de estudios}

La estrategia de búsqueda expuesta anteriormente arrojó 784 estudios (Figura 1). Se realizó una selección manual de los artículos. Los criterios de exclusión fueron: artículos no escritos en inglés, publicados en revistas no indexadas, artículos de revisión, no realizados en humanos o cadáveres humanos y aquellos no pertenecientes a la rodilla fueron excluidos. Dos investigadores (AGC, DGG) excluyeron artículos tras revisar título y abstract. El desacuerdo sobre los datos y la inclusión del estudio se resolvió mediante discusión y consenso con un autor principal.

\section{Resultados}

El número final de artículos seleccionados (132) fueron revisados para aclarar la incidencia, la anatomía, la biomecánica, la clínica, las pruebas de imagen, el tratamiento y la evolución del LAL. Los datos extraídos de los artículos fueron: tipo de estudio, fecha de publicación, autores, tamaño de la muestra, edad media, objetivo y resultados del mismo.

\section{Incidencia y anatomía}

El rango de prevalencia del citado ligamento en los diferentes estudios sobre especímenes cadavéricos humanos fue del $83-100 \%{ }^{(22)}$. Existe controversia en cuanto al origen extraarticular o intraarticular del mismo(8,9). Algunos reportan que la cápsula puede incorporar un número de fibras profundas del $\operatorname{LAL}^{(8,10,39)}$, mientras que otros defienden el origen extracapsular del mismo ${ }^{(9,10,12,22)}$.

La localización de la inserción femoral es la que más discusión provoca. En el año 2012, Vincent et al. ${ }^{(10)}$ realizaron 10 disecciones anatómicas de cadáver situando la inserción sobre el epicóndilo, anterior y distal al ligamento colateral lateral (LCL). En 2013, Claes et al. ${ }^{(1)}$ y Helito et al. ${ }^{(13)}$ situaron la inserción femoral de dicho ligamento anterior y distal al epicóndilo, anterior al LCL y proximal y posterior al tendón poplíteo en la mayoría de las 41 y 20 disecciones que realizaron, respectivamente. Al año siguiente, Dodds et al. ${ }^{(9)}$ realizaron 40 disecciones, encontrando la inserción del LAL proximal y posterior al epicóndilo y al LCL. En el año 2016, Daggett et al. ${ }^{(14)}$ localizaron en un estudio anatómico sobre 52 especímenes la inserción femoral con mayor frecuencia proximal y posterior al epicóndilo y LCL; posteriormente, de nuevo Claes $^{(15)}$ localiza la inserción en esta misma localización, no coincidiendo con su anterior publicación. Ese mismo 


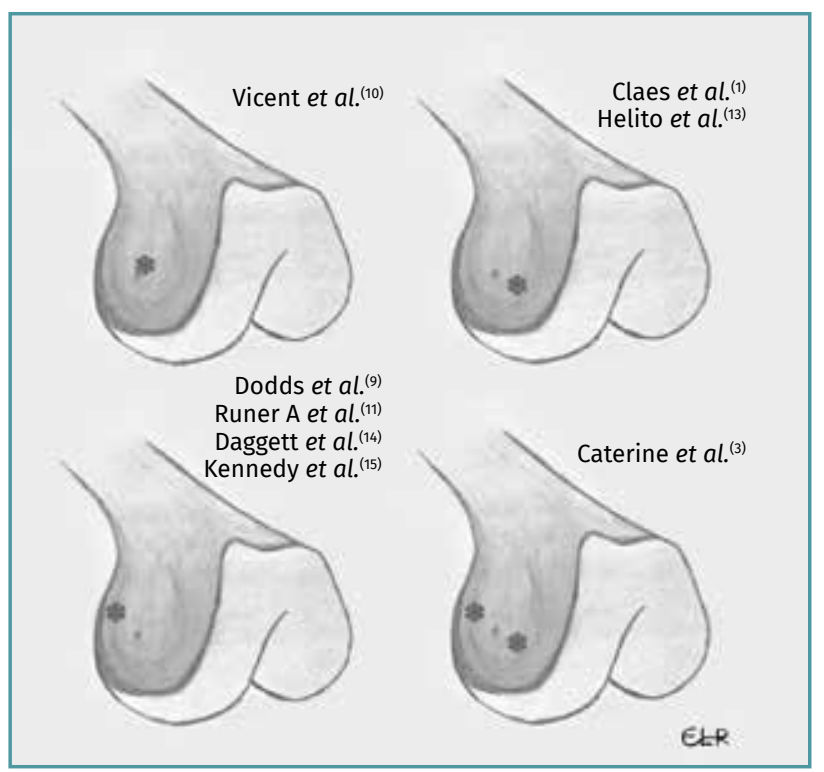

Figura 2. Origen femoral del ligamento anterolateral por los diferentes autores. podría explicar la asociación de lesiones del menisco externo asociadas al ligamento cruzado anterior $(\mathrm{LCA})^{(41)}$, presentes en el $44 \%$ de las reconstrucciones del $\mathrm{LCA}^{(42,43)}$, con lesión de la raíz meniscal en el $23 \%$ de esas lesiones ${ }^{(42)}$. Corbo et al. ${ }^{(41)}$ demostraron que las fibras inframeniscales eran más fuertes y rígidas en comparación con las suprameniscales, presentando ambas la misma composición histológica. Van Dyck ${ }^{(44)}$ et al. observaron que la mayoría de las lesiones se producian en la porción inframeniscal del LAL y que estos pacientes presentaban mayor riesgo de lesión meniscal lateral (61\%), en comparación con los que no presentaban lesión del LAL (31\%), sugiriendo que la lesión de estas fibras puede dar lugar a un menisco más móvil, soportando mayor tensión en la raíz posterior durante los episodios de subluxación anterolateral, provocando en consecuencia mayor riesgo de desgarro lateral del menisco. La inserción tibial ofrece menos dudas, localizándose en la mayoría de las ocasiones año se publica un estudio de disección anatómica sobre 44 rodillas de cadáver ${ }^{(11)}$, demostrando una inserción proximal y posterior al LCL. Catherine et al. ${ }^{(8)}$ comprueban una inserción variable, posterior y proximal al LCL o anterior y distal al mismo. Aunque Stijak et al. ${ }^{(12)}$ solo localizaron el ligamento en el $50 \%$ de las disecciones, su localización difiere de todas las anteriores. En todos los casos la unión del ligamento fue directamente al hueso ${ }^{(14)}$ (Figura 2).

El trayecto del ligamento ha sido descrito de diferentes maneras. Dodds et al. ${ }^{(9)}$ lo dirige en dirección oblicua (lateral a medial y proximal a distal) superfical al LCL; sin embargo, otros lo localizan profundo al $\mathrm{LCL}^{(1,10,12,13)}$, presentando una fuerte conexión con el tercio medio del menisco externo ${ }^{(1,8-12,14,39,40)}$, lo que

\section{Tabla 1. Prevalencia y localización del ligamento anterolateral (LAL) en los diferentes estudios}

\begin{tabular}{|c|c|c|c|c|}
\hline Autor & $\begin{array}{l}\text { N.o de disecciones } \\
\text { anatómicas }\end{array}$ & Prevalencia & & Localización origen LAL \\
\hline \multirow{2}{*}{ Vicent $^{(10)}$} & \multirow{2}{*}{10} & \multirow{2}{*}{$10(100 \%)$} & 9 & $\begin{array}{l}\text { Sobre el epicóndilo } \\
\text { Anterior al tendón poplíteo }\end{array}$ \\
\hline & & & 1 & $\begin{array}{l}\text { Sobre el epicóndilo } \\
\text { Distal al tendón poplíteo }\end{array}$ \\
\hline Claes $^{(1)}$ & 41 & $41(100 \%)$ & \multicolumn{2}{|c|}{$\begin{array}{l}\text { Anterior y distal al epicóndilo } \\
\text { Proximal y posterior al tendón poplíteo }\end{array}$} \\
\hline Helito $^{(13)}$ & 20 & $20(100 \%)$ & \multicolumn{2}{|c|}{$\begin{array}{l}\text { Distal y anterior al ligamento colateral } \\
\text { lateral }(\mathrm{LCL})\end{array}$} \\
\hline Dodds $^{(9)}$ & 40 & $33(83 \%)$ & \multicolumn{2}{|c|}{ Proximal y posterior al epicóndilo } \\
\hline \multirow{2}{*}{ Daggett $^{(14)}$} & \multirow{2}{*}{52} & \multirow{2}{*}{$52(100 \%)$} & 12 & Sobre el epicóndilo \\
\hline & & & 40 & Posterior y proximal al epicóndilo \\
\hline \multirow[b]{2}{*}{ Kennedy ${ }^{(15)}$} & \multirow[b]{2}{*}{15} & \multirow[b]{2}{*}{$15(100 \%)$} & 14 & Posterior y proximal a la unión $\mathrm{LCL}$ \\
\hline & & & 1 & $\begin{array}{l}\text { Sobre epicóndilo } \\
\text { Posterior y proximal al LCL }\end{array}$ \\
\hline \multirow{2}{*}{ Runer $\mathbf{A}^{(11)}$} & \multirow{2}{*}{44} & \multirow{2}{*}{$20(45,5 \%)$} & 9 & Sobre el epicóndilo \\
\hline & & & 11 & Proximal y posterior al epicóndilo. \\
\hline \multirow{2}{*}{ Caterine $^{(8)}$} & \multirow{2}{*}{19} & \multirow{2}{*}{$19(100 \%)$} & 11 & Anterior y distal a LCL \\
\hline & & & 8 & Posterior y proximal a $\mathrm{LCL}$ \\
\hline Stijak ${ }^{(12)}$ & 14 & $7(50 \%)$ & \multicolumn{2}{|c|}{ Sobre el epicóndilo } \\
\hline
\end{tabular}




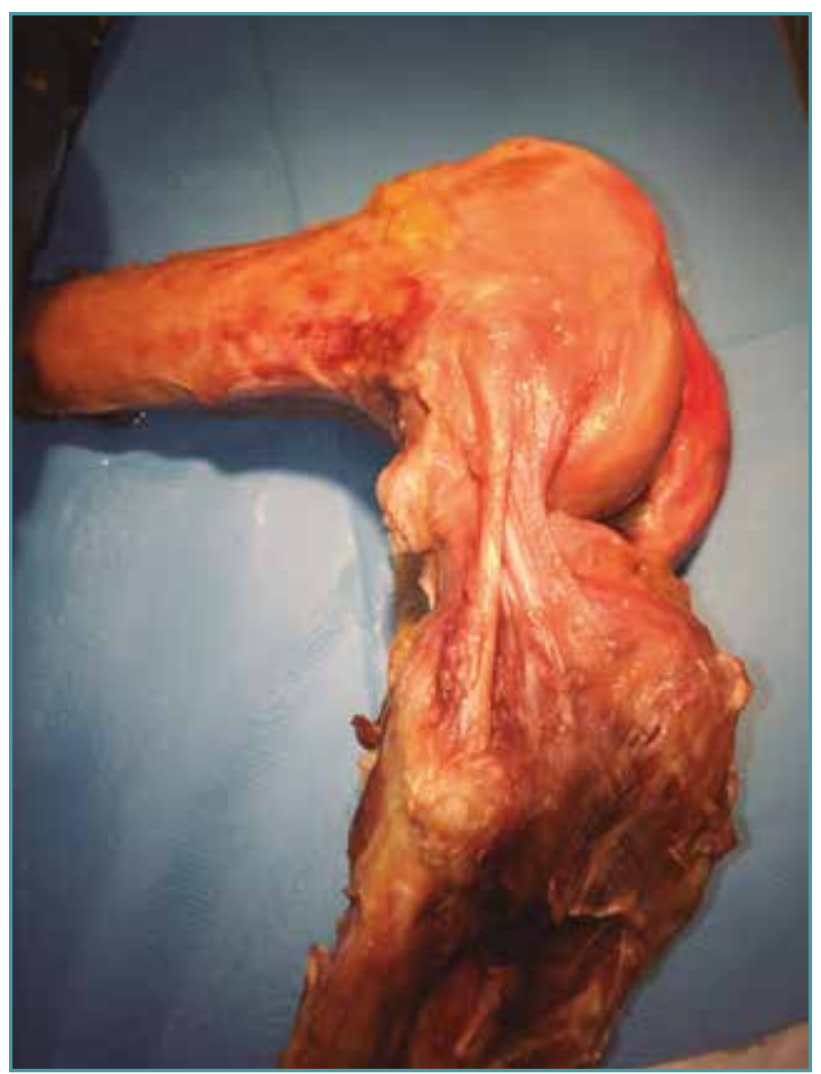

Figura 3. Disección anatómica de una rodilla humana derecha. Se aprecia la presencia del ligamento anterolateral (LAL) que discurre desde una inserción amplia en la región comprendida entre el tubérculo de Gerdy y la cabeza del peroné, pasa por debajo del ligamento colateral lateral $(L C L)$ y se inserta en las proximidades del la inserción femoral del LCL, proximal y posterior a este.

posterior al tubérculo de Gerdy ${ }^{(1)}$, en la mitad entre una línea que une la cabeza del peroné con el tubérculo de Gerdy ${ }^{(1,8-11,39,40)}$ (Tabla 1 y Figura 3).

La inserción femoral tenía una anchura media de 7,2-11,85 mm $\mathrm{m}^{(1,11,14)}$, mientras que la tibial de 11,2$12,2 \mathrm{~mm}^{(1,11)}$. Las dimensiones del mismo son de 35 a $45 \mathrm{~mm}$ de longitud, $2 \mathrm{~mm}$ de anchura y de 1 a $2 \mathrm{~mm}$ de grosor ${ }^{(1,8-10)}$, siendo tanto la longitud como el grosor mayores en varones que en mujeres $^{(11,45)}$. No se han apreciado diferencias en el peso del ligamento con respecto al sexo ${ }^{(45)}$.

\section{Biomecánica}

En los últimos años existe mucho interés en las estructuras anterolaterales y la contribución de cada componente en la estabilidad rotacional de la rodilla.

En los años setenta, Hughston et al.(3), al describir el compartimento lateral de la rodilla, concluyeron que el "midthird capsular ligament" es el principal estabilizador lateral estático a $30^{\circ}$ de flexión. En el año 2012, Monaco ${ }^{(23)}$ et al. observaron en 10 rodillas cadavéricas intactas que la sección del LCA produce un leve aumento de la rotación interna, mientras que la sección de las estructuras anterolaterales produce un aumento significativo en todos los grados de flexión de la rodilla, sobre todo a $30^{\circ}$, contribuyendo también a la estabilidad anteroposterior. En su estudio inicial, Claes et al.(1) observaron que el LAL se encuentra completamente tensionado en flexión y rotación interna. En el estudio biomecánico de Ruiz et al. ${ }^{(16)}$, se demostró que se produce un aumento de la rotación interna a $30^{\circ}$ tras la sección del LCA y sobre todo de las estructuras anterolaterales, aumentando esta rotación si se producía la sección de ambos. Estos resultados estaban en consonancia con el estudio de Spencer et al. ${ }^{(18)}$. El orden en que se realizaba la sección no influyó en la rotación resultante, por lo que se podía deducir que ambos ligamentos eran independientes y sinérgicos.

Parece ser que la localización femoral del LAL influye en la isometría del mismo. Zens et al. ${ }^{(19)} \mathrm{y}$ Kittl et al. ${ }^{(46)}$ realizaron estudios biomecánicos en cadáver teniendo en cuenta la localización definida por Claes $^{(1)}$, anterior y distal al epicóndilo, y observaron un aumento de la longitud de manera uniforme desde la extensión completa hasta los $90^{\circ}$ de flexión. Las rotaciones modificaban la longitud, aumentando la longitud en el caso de la rotación interna y disminuyendo en la externa.

Un estudio reciente realizado sobre 12 cadáveres $^{(47)}$ teniendo en cuenta las 3 localizaciones femorales más frecuentes reflejadas antes en la anatomía (epicóndilo, proximal y posterior a este, y distal y anterior al epicóndilo) observó que durante la rotación interna se produce un incremento de la longitud para las 3 localizaciones femorales a $20^{\circ}$, pero estaban significativamente aumentadas a $90^{\circ}$. La mayor longitud fue observada para la inserción posterior y proximal al epicóndilo y la menor para la anterior y distal al mismo. En este mismo estudio se comprobó que, si se mantiene la pierna en rotación neutra, se produce una disminución de la longitud en la 
localización proximal y posterior, y un aumento de la misma tanto en la posición anterior y distal como la situada sobre el epicóndilo. Si tenemos en cuenta que, en los primeros estudios biomecánicos ${ }^{(1,8-10,13,16,17)}$, la función del LAL era el control de la rotación interna cerca de la extensión completa de la rodilla, podemos deducir que la localización posterior y proximal es la más isométrica teniendo un rango articular completo.

Los estudios biomecánicos más recientes ponen de manifiesto que el LAL es un estabilizador primario de la rotación interna de la rodilla a $35^{\circ}$ o más de flexión con un mínimo rol en la estabilización anteroposterior $r^{(15,18-21)}$. Dodds et al. ${ }^{(9)}$ detectaron que el LAL es isométrico desde la extensión completa hasta la flexión de $60^{\circ}$, mientras que entre $60^{\circ}$ y $90^{\circ}$ apreciaron un acortamiento significativo, teniendo en cuenta su localización proximal y posterior al epicóndilo y la rotación neutra del miembro. Las rotaciones no producen cambios en la longitud con la rodilla en extensión completa, mientras que al pasar de $30^{\circ}$ grados de flexión se produce un alargamiento del mismo, más significativo a $90^{\circ}$. Al realizar la rotación externa con la rodilla en flexión, se acorta. Estos hallazgos también fueron observados por Lutz et al. ${ }^{(20)}$. Helito et al. ${ }^{(48)}$ realizaron un estudio mediante tomografía computarizada para registrar los cambios de longitud en los diferentes grados de flexión y rotación de la rodilla, apreciando un incremento de la longitud desde los $0^{\circ}$ a los $90^{\circ}$ presentando el mayor crecimiento desde los $60^{\circ}$ a los $90^{\circ}$. En 2015, Parsons et al. ${ }^{(24)}$, en un estudio biomecánico in vitro realizado sobre 11 rodillas cadavéricas, observaron que la contribución rotacional del LAL y el LCA a $30^{\circ}$ eran similares, pero a medida que aumentábamos la flexión, la estabilización por el LAL era mayor, no teniendo ninguna función en la traslación anteroposterior de la tibia. Sonnery Cottet et al. ${ }^{(49)}$ destacan el papel del LAL en el control de la rotación interna a $90^{\circ}$ de flexión pero no a $20^{\circ}$, actuando conjuntamente con la banda iliotibial. Rasmussen et al. ${ }^{(17)}$ demostraron biomecánicamente en un estudio robótico que la lesión conjunta del LCA y el LAL provoca una mayor inestabilidad rotacional en comparación con la lesión única del LCA desde los $0^{\circ}$ a los $120^{\circ}$ de flexión. Sin embargo, otros autores ${ }^{(50-53)}$ defienden el papel secundario del LAL en el control de la rotación interna en rodillas con deficiencia del LCA, siendo el tracto iliotibial el principal restrictor a la rotación interna, aumentando su contribución a medida que aumentaba la flexión ${ }^{(49,50)}$. En un reciente estudio biomecánico in vitro sobre 10 rodillas cadavéricas, Bonanzinga et al. ${ }^{(54)}$ comprobaron que el LAL no influye en el control de la estabilidad anteroposterior, siendo el LCA el principal estabilizador tanto a $30^{\circ}$ como a $90^{\circ}$. Sin embargo, sí se apreciaba un aumento de la rotación interna tanto a $30^{\circ}$ como $90^{\circ}$ tras la sección del LAL y un aumento en la aceleración del pivot shift test.

Puede ser que se haya infravalorado el papel de la banda iliotibial en el control de la rotación interna de la rodilla debido al enfoque en el LAL de los últimos años, tal y como aprecian Yamamoto ${ }^{(55)}$ et al. y Hassler y Jacob ${ }^{(56)}$, donde la resección de la banda iliotibial provoca un gran incremento de la rotación interna.

\section{Clínica}

La parte fundamental para un buen tratamiento de las lesiones ligamentosas de la rodilla es realizar un correcto diagnóstico. Dichas lesiones provocan cambios en la biomecánica de la misma favoreciendo su inestabilidad y su degeneración. Estos cambios en la estabilidad pueden ser identificados en la exploración física con los test específicos (anteroposterior Lachman test, varo-valgo stress test, dial test...); sin embargo, las exploraciones de otras estructuras ligamentosas, como el LAL, raíces meniscales, etc., no están bien establecidas.

Los estudios biomecánicos sobre el LAL más recientes ponen de manifiesto el control de la estabilidad rotacional a grandes ángulos de flexión $n^{(9,18,20,21,24,47-49)}$, donde no existiría un pivot shift significativo (se explora a unos $30^{\circ}$ de flexión), mientras que otros defienden el control del mismo en extensión y escasos ángulos de fle$x_{i o ́} n^{(1,8-10,13,16,17)}$, con posibilidad de ser apreciado en el pivot shift, siendo un restrictor secundario en la estabilidad anteroposterior.

La maniobra de pivot shift nos muestra una inestabilidad rotacional, existiendo debate entre las estructuras cuya insuficiencia es responsable de su aparición (LCA, LAL, banda iliotibial, raíz del menisco externo...) y el grado en que contribuye cada una ${ }^{(4,23,50,57-61)}$. Así, varios autores han intentado esclarecer este concepto, analizando la alteración del LAL con el grado de pivot shift aprecia- 
do, demostrando que un alto grado de pivot shift está relacionado con la lesión de dicho ligamento ${ }^{(22-26)}$. Song et al. ${ }^{(62)}$ reportaron que, en el 78,7\% de las lesiones del LAL objetivadas mediante RM en lesiones concomitantes al LCA, se apreciaba un pivot shift de grado II-III, al igual que en otras publicaciones ${ }^{(18,63,64)}$.

Sin embargo, otros estudios ${ }^{(50,51,54,65)}$, en los que se secciona el $L A L$, no encuentran un aumento significativo del pivot shift con un LCA intacto, obteniendo un mayor aumento de la rotación interna a $60^{\circ}$ de flexión.

Ferreti et al. ${ }^{(66)}$, en su estudio, analizaron el tipo de lesión en el compartimento lateral hallada durante la cirugía con el grado de pivot shift presente, sin obtener correlación entre el tipo de lesión y el grado de pivot shift encontrado; no obstante, en todos los casos con pivot shift de grado II o III, la reconstrucción ligamentosa del mismo reducía o hacía desaparecer el pivot shift.

\section{Pruebas complementarias}

\section{Radiología simple}

En 1879, Paul Segond describió una fractura-avulsión en la parte proximal externa de la tibia con la rotación interna forzada en rodillas cadavéricas humanas ${ }^{(2)}$ debido a una banda fibrosa y resistente. Cien años después de la descripción de esta lesión, en 1979, se comenzó a asociar esta lesión con la presencia de inestabilidad en la rodilla ${ }^{(67)}$.

Clásicamente, se ha considerado a la fractura de Segond un signo patognomónico de la lesión del LCA en la población adulta ${ }^{(68-70)}$. La localización de la inserción tibial del LAL posterior al tubérculo de Gerdy ${ }^{(1,8-11,39,40)}$ y la frecuente combinación con la lesión del LCA hacen que se haya relacionado la lesión de esta estructura con la fractura de Segond $^{(9,27,28)}$. La banda iliotibial ${ }^{(5,68,69)}$, la zona central de la cápsula articularr(6,67), el brazo anterior de la cabeza corta del bíceps femoral(70) y la banda anterior del $\mathrm{LCL}^{(5,71)}$ también han sido propuestos como causantes de esta lesión ${ }^{(72)}$ (Figura 4).

\section{Ecografia}

La ultrasonografía es una herramienta de diagnóstico fiable para identificar una estructura hi-

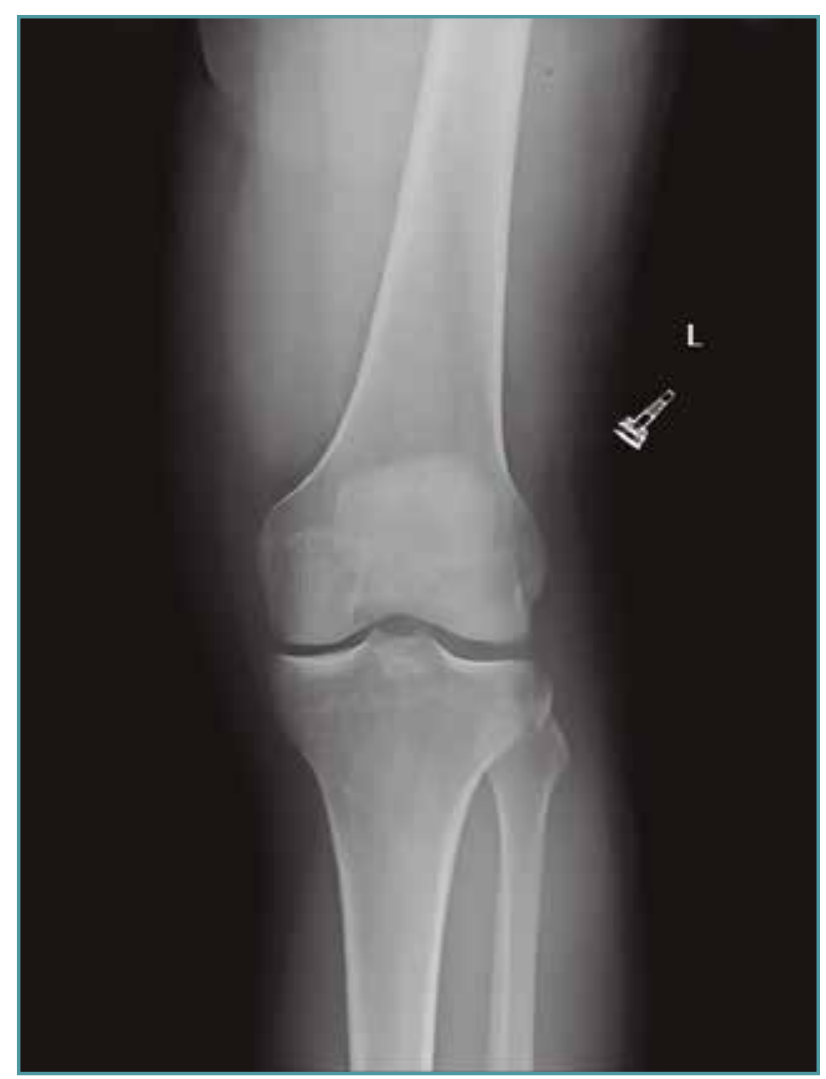

Figura 4. Avulsión ósea en zona lateral de tibia proximal (fractura de Segond).

perecogénica en la zona anterolateral de la rodilla correspondiente al LAL ${ }^{(31-33)}$. Además, permite un estudio dinámico de la misma. La fractura de Segond también ha sido diagnosticada en un alto porcentaje de las rodillas analizadas en comparación con la radiología simple o la $\mathrm{RM}^{(31,73)}$, lo cual puede ser explicado por la posición en la que la exploración es realizada.

\section{Resonancia magnética}

La visualización del LAL mediante RM para poder realizar un diagnóstico conjunto con lesiones del LCA también ha sido estudiada. El primer problema que se encontró fue la dificultad para la identificación de este ligamento con los protocolos habituales de $\mathrm{RM}^{(29-74)}$. Helito et al. ${ }^{(29)}$ visualizaron el LAL en el $97 \%$ de los casos con una máquina de 1,5 T y cortes de 2,5 y $3 \mathrm{~mm}$. Caterine et al. ${ }^{(8)}$ pudieron apreciar las características de este ligamento en las 10 rodillas estudiadas, al igual que Porrino 
et al. ${ }^{(28)}$, utilizando los primeros una maquina de $3 \mathrm{~T}$ y con cortes de 0,4 mm. Por su parte, Kosy et al. ${ }^{(30)}$ identificaron la citada estructura en el $94 \%$ de las 100 rodillas estudiadas. Sin embargo, Taneja et al. ${ }^{(75)}$ solamente pudieron observar la estructura en el $51 \%$ de las RM con un grosor de cortes de $3 \mathrm{~mm}$.

Los estudios anteriores visualizan un ligamento sin alteraciones, aunque también ha sido estudiada mediante RM la posible afectación del mismo asociado a lesiones del $\mathrm{LCA}^{(44,76,77)}$. La prevalencia de lesiones asociadas en el LAL cuando se encuentra el LCA roto varía entre 39,5 y 78,7\% con mayor afectación de la inserción tibial del $\operatorname{LAL}^{(44,76)}$, aunque Helito et al. ${ }^{(77)}$ localizan por igual la lesión tanto en zona proximal como distal.

También se ha encontrado correlación entre disecciones anatómicas y estudio por RM(78).

Por el contrario, otros autores visualizan el LAL con una resonancia de 1,5 T y cortes de $4 \mathrm{~mm}$, pero no con suficiente detalle para determinar si está alterado o intacto debido a sus dimensiones ${ }^{(79)}$, al igual que sucede en el estudio de Devitt et al. ${ }^{\left({ }^{80}\right)}$, en el que utilizando una resonancia de $3 \mathrm{~T}$ no consiguen identificar en la mayoría de los casos la completa longitud del LAL tanto en rodillas sanas como en las que presentan rotura del LCA. En otro estudio no se consigue visualizar debido a la confluencia con el LCL y la banda iliotibial(28).

El análisis de la fractura de Segond también ha sido realizado por RM en diferentes estudios, aunque la prevalencia de la misma con esta prueba complementaria, al igual que con la radiología simple, es baja ${ }^{(44,76)}$. En estudios recientes ${ }^{(44,76)}$ se ha observado que la fractura de Segond solamente ha podido visualizarse en el $1,8 \%$ de las lesiones del LAL en uno de los estudios ${ }^{(76)}$, en el $6,1 \%$ en otro ${ }^{(77)} \mathrm{y}$ en el $37 \%$ en el restante ${ }^{(44)}$.

\section{Tratamiento}

El tratamiento de la inestabilidad anterolateral de la rodilla, puesto de manifiesto con la prueba diagnóstica del pivot shift, ha ido evolucionando a lo largo del tiempo con el objetivo de un mejor control del mismo. Hace varias décadas se comenzó a utilizar la tenodesis extraarticular aislada(81-84), controlando de forma efectiva la inestabilidad rotacional pero ejerciendo solamente un moderado control sobre la inestabilidad anteroposterior con resultados pobres a largo plazo. Además, se ob-

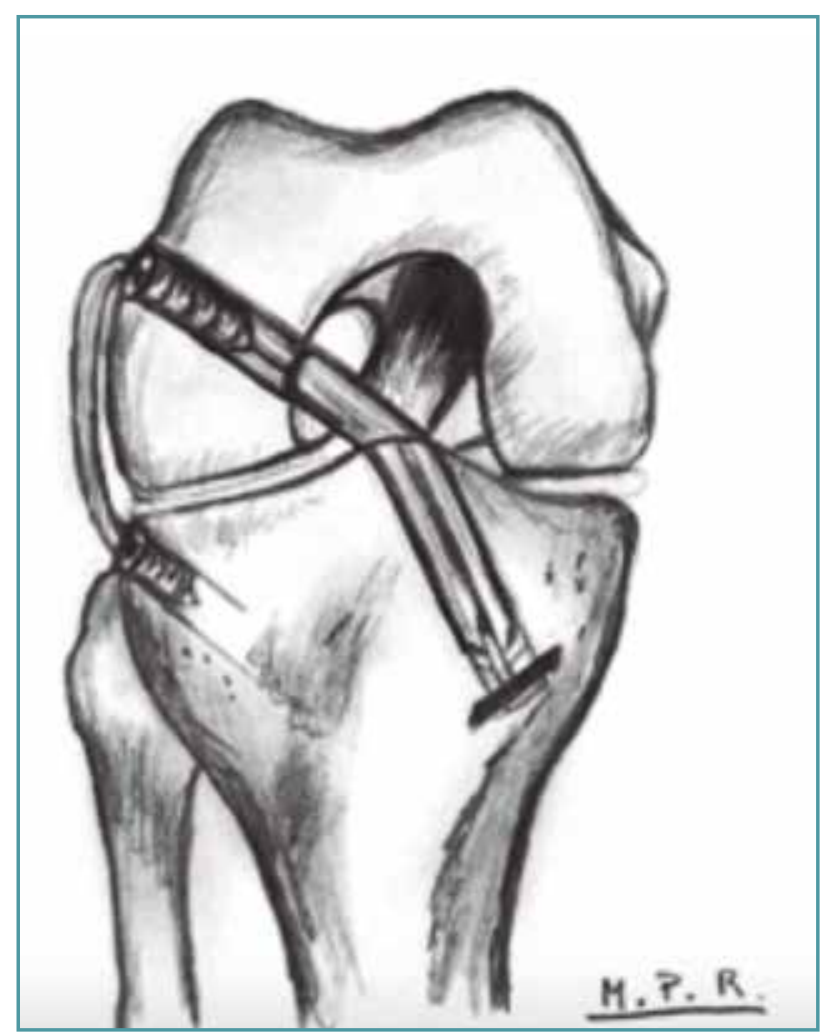

Figura 5. Reconstrucción del ligamento cruzado anterior (LCA) asociada a refuerzo lateral extraarticular.

servó un aumento de cambios degenerativos en el compartimento lateral de la rodilla(85,86).

Posteriormente, se pasó a la reconstrucción intraarticular transtibial del LCA mediante fascículo único, convirtiéndose en el gold estándar, con una orientación más vertical de la misma, mejorando en este caso la inestabilidad anteroposterior, pero con resultados pobres en el control de la inestabilidad rotacional ${ }^{(87,88)}$.

En un afán de mejorar este control rotacional se comenzaron a realizar reconstrucciones con doble fascículo del LCA ${ }^{(89-91)}$. Los estudios biomecánicos comparativos entre ambas técnicas demostraron la superioridad de la doble banda ${ }^{(92,93)}$, no apreciándose diferencias en cuanto a la estabilidad y la progresión a artrosis con respecto a la banda única, pero sí se pudo comprobar un menor indice de rotura de la plastia, con un periodo de seguimiento de 10 años ${ }^{(94,95)}$. Otros inconvenientes con este tipo de intervención son la mayor dificultad técnica y los problemas en la cirugía de revisión de la misma, por lo que hoy en día ha perdido popularidad. 


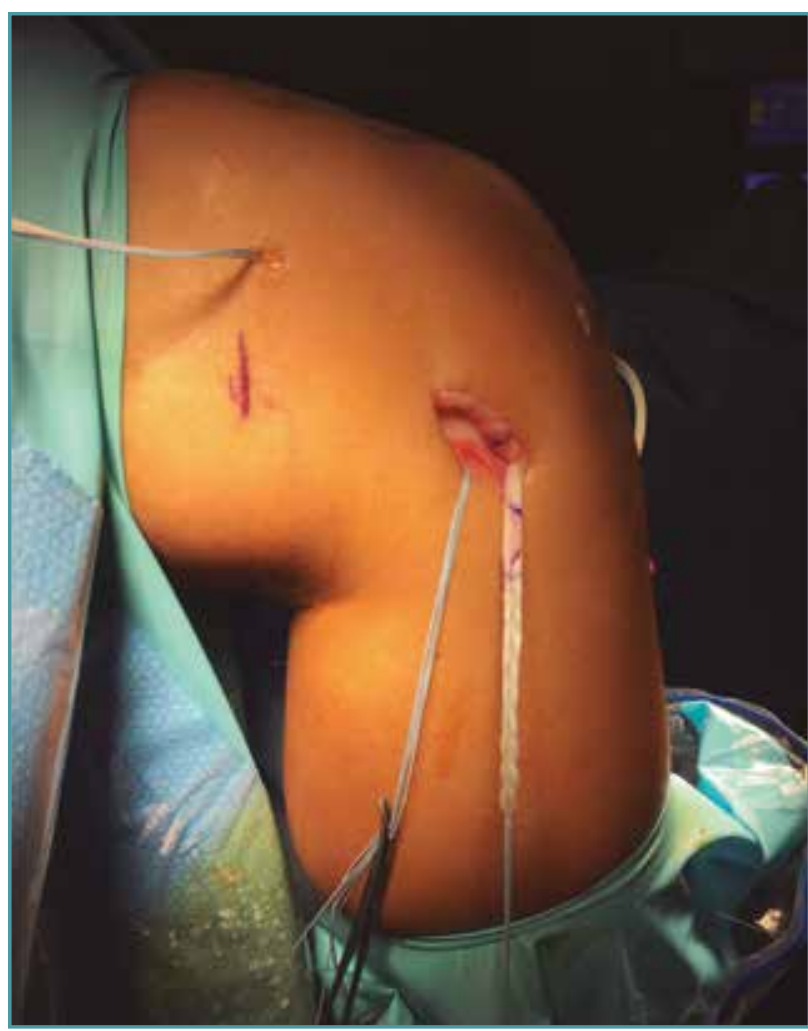

Figura 6. Imagen quirúrgica de una tenodesis extraarticular lateral de tipo Lemaire modificada. Nótese la presencia de los hilos de tracción de la plastia intraarticular anatómica del ligamento cruzado anterior (LCA).

Actualmente el gold estándar es la reconstrucción del LCA con banda única en posición anatómica ${ }^{(96,97)}$, demostrando unos resultados satisfactorios en la mayoría de los $\operatorname{casos}^{(98)}$. A pesar de esto, en algunos casos persiste la inestabilidad rotacional, presentando los pacientes un pivot shift positivo tras la reconstrucción ${ }^{(99)}$, relacionándose con peores resultados ${ }^{(100)}$.

Hoy en día, con el objetivo de controlar este pivot shift residual, se está asociando en algunos pacientes técnicas de reconstrucción de las estructuras anterolaterales asociadas al LCA, usando diferentes estructuras como son la fascia lata(34), tendones de la pata de ganso ${ }^{(35)}$, aloinjertos ${ }^{(36)} \mathrm{e}$ incluso injertos artificiales ${ }^{(37)}$ (Figura 5).

Para conseguir una reconstrucción exitosa, la posición de la plastia es fundamental(23) (Figura 6). La malposición de los túneles y los cambios en la longitud con respecto al grado de flexión de la rodilla pueden llevar a un aumento de tensión en el compartimento lateral y la plastia, lo que degenera en un fallo de la plastia y degeneración en el compartimento lateral ${ }^{(19,23)}$.

La localización femoral del citado ligamento es la más discutida ${ }^{(1,3,4,5,7,7,8,1,0,11)}$. En un estudio reciente, Kittl et al. ${ }^{(46)}$ desarrollaron un estudio biomecánico registrando las variaciones en la longitud del ligamento utilizando las diferentes localizaciones del mismo con ángulos de flexión de $0^{\circ}$ a $90^{\circ}$ y determinan que la posición posterior y proximal al epicóndilo, pasando profundo al LCL y con fijación en extensión completa, era la más isométrica. Sin embargo, Katakura et al. ${ }^{(101)}$ refieren que no hay diferencia entre pasar el injerto superficial o profundo al LCL.

Con respecto al tipo de injerto utilizado para la reconstrucción, la banda iliotibial tiene mejores propiedades biomecánicas en comparación con el tendón del gracilis ${ }^{(102,103)}$.

Estudios recientes demuestran una disminución del pivot shift asociando esta reconstrucción del LAL a la cirugía del LCA ${ }^{(104-106)}$. Mónaco et al. ${ }^{(107)}$ demostraron una disminución del pivot shift con la utilización de una reconstrucción anterolateral asociada a la del LCA en comparación con la técnica de doble banda del LCA. La utilización de este refuerzo en cirugías de revisión del LCA también mejora la estabilidad de la rodilla(106).

Sin embargo, Anderson et al. ${ }^{(108)}$ y Roth et al. ${ }^{(109)}$ no encontraron ningún beneficio al añadir este refuerzo lateral, al igual que otro estudio actual(110).

Sonnery-Cottet et al. ${ }^{(38)}$, en un estudio clínico prospectivo con 2 años de evolución, evidenciaron mejoría en los pacientes con esta plastia de refuerzo lateral tanto en estabilidad como en fallos y complicaciones.

\section{Discusión}

El control rotacional anterolateral de la rodilla es esencial para la estabilidad y la función de la misma. Clásicamente, ha sido considerado una parte fundamental del papel biomecánico del $\mathrm{LCA}^{(111)}$, sobre todo del fascículo posterolateral del mismo; sin embargo, en los últimos tiempos su papel ha sido cuestionado ${ }^{(112)}$ y otras estructuras han sido estudiadas para conocer su función en esta inestabilidad ${ }^{(4,23,50,57-61)}$.

A pesar de que la mayoría de las reconstrucciones del LCA consiguen restaurar la normal traslación anteroposterior femorotibial, existe un 
elevado porcentaje de pacientes en los que persiste esta inestabilidad rotacional al no controlar el pivot shift(38,99,100).

Las técnicas quirúrgicas, el tejido empleado y los dispositivos de anclaje han ido evolucionando con el paso del tiempo en un intento de mejorar la función y controlar esta inestabilidad residual en algunos pacientes. Así, en 1916 se realizó la primera reconstrucción del LCA por Hey Groves de Bristol; posteriormente se pasó al uso de técnicas extraarticulares ${ }^{(81-84)}$, no consiguiendo el control deseado en la estabilidad anteroposterior.

En las décadas de los ochenta y noventa se generalizó el uso de reconstrucciones intraarticulares, realizando en 1980 la primera reconstrucción artroscópica por David Dandy de Cambridge.

La técnica tradicional de 2 incisiones dio paso a una única incisión, realizando el túnel femoral a través del túnel tibial (técnica transtibial); sin embargo, algunos autores comunicaron la no posibilidad de colocar el injerto en posición anatómica, obteniendo una orientación más vertical de la misma ${ }^{(13,114)}$. Por lo que se pasó a dar una orientación más oblicua a la plastia para mejorar el control de la rotación, situándola en posición de las 10 horas en lugar de las 11 horas ${ }^{(115)}$, desde el portal anteromedial.

En un afán de mejorar la estabilidad proporcionada y restaurar la original anatomía del LCA, se pasó a técnicas de reconstrucción bifascicu$\operatorname{lar}^{(89-91)}$, pero fueron abandonadas por no encontrar mejoría clínica con respecto a las monofasciculares $^{(94,95)}$ y ser técnicamente más demandantes, con peores soluciones en las cirugías de revisión.

Quizás porque la anatomía es demasiado compleja ${ }^{(116-118)}$, es probable que sea imposible reproducir la anatomía fiablemente para que reproduzca la función del ligamento.

Por otro lado, la existencia de las estructuras anterolaterales es evidente ${ }^{(22)}$ y su papel en el control de la rotación ha sido demostrado. Clásicamente, se pensaba que limitaba la rotación interna en grados cercanos a la extensión ${ }^{(8-10,16,17) ;}$;in embargo, otros estudios ponen de manifiesto una limitación rotacional a grados altos de flexión de la rodilla ${ }^{(15,19,21,49)}$.

Dada la importancia del LAL en el control del pivot shift ${ }^{(62-64,66)}$, se ha retomado el interés en las reconstrucciones de estas estructuras asociadas a la cirugía del LCA en un intento de disminuir el pivot shift residual y mejorar la estabilidad.
Recientemente, se ha publicado un estudio sobre la evolución de pacientes con reconstrucción anterolateral con buenos resultados ${ }^{(38)}$, por lo que dejando a un lado la existencia o no del citado ligamento y la función del mismo, si se aprecia menor pivot, mejor función y menor índice de rerroturas, parece ser una buena opción de tratamiento. Actualmente, se encuentra en curso un ensayo clínico sobre la evolución de estos pacientes y podrá aclarar un poco más esta cuestión ${ }^{(119)}$.

Aun así, con resultados prometedores en la reconstrucción anatómica del LAL, los resultados son a muy corto plazo, por lo que se deberían evaluar a más largo plazo, con estudios comparativos incluyendo las distintas variantes anatómicas femorales y comparando los resultados de reconstrucción del LCA junto al refuerzo lateral con la reconstrucción aislada del LCA.

\section{Conclusiones}

- La existencia del LAL de la rodilla es evidente.

- Su función principalmente es el control de la rotación interna, según parece, en grados cercanos a la extensión completa.

- Para su diagnóstico se dispone de la maniobra de pivot shift en la exploración, la fractura de Segond en la radiografía simple y otras pruebas complementarias como la ecografía y la RM.

- La reconstrucción de esta estructura obtiene resultados satisfactorios.

\section{Responsabilidades éticas}

Conflicto de interés. Los autores declaran no tener ningún conflicto de intereses.

Financiación. Este trabajo no ha sido financiado.

Protección de personas y animales. LOS autores declaran que para esta investigación no se han realizado experimentos en seres humanos ni en animales.

Confidencialidad de los datos. Los autores declaran que han seguido los protocolos de su centro de trabajo sobre la publicación de datos de pacientes.

Derecho a la privacidad y consentimiento informado. Los autores declaran que en este artículo no aparecen datos de pacientes. 


\section{Bibliografía}

1. Claes S, Vereecke E, Maes M, Victor J, Verdonk P, Bellemans J. Anatomy of the anterolateral ligament of the knee. J Anat. 2013;223(4):321-8.

2. Segond P. Recherches cliniques et expérimentales sur les épanchements sanguins du genou par entorse. Prog Med (Paris) 1879:1-85. Disponible en: http://www. patrimoine.edilivre.com.

3. Hughston JC, Andrews JR, Cross MJ, Moschi A. Classification of knee ligament instabilities. Part II. The lateral compartment. J Bone Joint Surg Am. 1976;58(2):173-9.

4. Terry GC, Norwood LA, Hugston JC, Cladwell KM. How iliotibial tract injuries of the knee combine with acute anterior cruciate ligament tears to influence abnormal anterior tibial displacement. Am J Sports Med. 1993;21(1):55-60.

5. Campos JC, Chung CB, Lektrakul N, Pedowitz R, Trudell D, Yu J, Resnick D. Pathogenesis of the Segond fracture: anatomic and MR imaging evidence of an iliotibial tract or anterior oblique band avulsion. Radiology. 2001;219(2):381-6.

6. Johnson LL. Lateral capsular ligament complex: Anatomical and surgical considerations. Am J Sports Med. 1979;7(3):156-60.

7. Vieira EL, Vieira EA, da Silva RT, Berlfein PA, Abdalla RJ, Cohen M. An anatomic study of the iliotibial tract. Arthroscopy. 2007;23:269-74.

8. Caterine S, Litchfield R, Johnson M, Chronik B, Getgood A. A cadaveric study of the anterolateral ligament: re-introducing the lateral capsular ligament. Knee Surg Sports Traumatol Arthrosc. 2015;23(11):3186-95.

9. Dodds AL, Halewood C, Gupte CM, Williams A, Amis AA. The anterolateral ligament: anatomy, length changes and association with the Segond fracture. Bone Joint J. 2014;96-B(3):325-31.

10. Vincent JP, Magnussen RA, Gezmez F, Uguen A, Jacobi $M$, Weppe $F$, et al. The anterolateral ligament of the human knee: an anatomic and histologic study. Knee Surg Sports Traumatol Arthrosc. 2012;20(1):147-52.

11. Runer A, Birkmaier S, Pamminger M, Reider S, Herbst E, Künzel KH, et al. The anterolateral ligament of the knee: a dissection study. Knee. 2016;23(1):8-12.

12. Stijak L, Bumbasirevic M, Radonjic V, Kadija M, Puskas L, Milovanovic D, Filipovic B. Anatomic description of the anterolateral ligament of the knee. Knee Surg Sports Traumatol Arthrosc. 2016;24(7):2083-8.

13. Helito CP, Demange MK, Bonadio MB, Tirico LE, Gobbi RG, Pécora JR, Camanho GL. Anatomy and histology of the knee anterolateral ligament. Orthop J Sports Med. 2013;1(7):2325967113513546.
14. Daggett M, Ockuly AC, Cullen M, Busch K, Lutz C, Imbert $\mathrm{P}$, Sonnery-Cottet $\mathrm{B}$. Femoral origin of the anterolateral ligament: an anatomic analysis. Arthroscopy. 2016;32(5):835-41.

15. Kennedy MI, Claes S, Fuso FA, Williams BT, Goldsmith MT, Turnbull TL, et al. The anterolateral ligament: an anatomic, radiographic, and biomechanical analysis. Am J Sports Med. 2015;43(7):1606-15.

16. Ruiz N, Filippi GJ, Gagnière B, Bowen M, Robert HE. The comparative role of the anterior cruciate ligament and anterolateral structures in controlling passive internal rotation of the knee: a biomechanical study. Arthroscopy. 2016;32(6):1053-62.

17. Rasmussen MT, Nitri M, Williams BT, Moulton SG, Cruz RS, Dornan GJ, et al. An in vitro robotic assesment of the anterolateral ligament, Part 1: Secondary role of the anterolateral ligament in the setting of an anterior cruciate ligament injury. Am J Sports Med. 2016;44(3): 585-92.

18. Spencer L, Burkhart TA, Tran MN, Rezansoff AJ, Deo S, Caterine S, Getgood AM. Biomechanical analysis of simulated clinical testing and reconstruction of the anterolateral ligament of the knee. Am J Sports Med. 2015;43(9):2189-97.

19. Zens M, Niemeyer P, Ruhhammer J, Bernstein A, Woias $\mathrm{P}$, Mayr $\mathrm{HO}$, et al. Length changes of the anterolateral ligament during passive knee motion: a human cadaveric study. Am J Sports Med. 2015;43(10):2545-52.

20. Lutz C, Sonnery-Cottet B, Niglis L, Freychet B, Clavert $\mathrm{P}$, Imbert P. Behavior of the anterolateral structures of the knee during internal rotation. Orthop Traumatol Surg Res. 2015;101(5):523-8.

21. Drews BH, Kessler O, Franz W, Dürselen L, Freutel M. Function and strain of the anterolateral ligament part I: biomechanical analysis. Knee Surg Sports Traumatol Arthrosc. 2017;25(4):1132-9.

22. Pomajzl R, Maerz T, Shams C, Guettler J, Bicos J. A review of the anterolateral ligament of the knee: current knowledge regarding its incidence, anatomy, biomechanics, and surgical dissection. Arthroscopy. 2015;31(3):583-91.

23. Monaco E, Ferreti A, Labianca L, Maestri B, Speranza A, Kelly MJ, D’Arrigo C. Navigated knee kinematics after cutting of the ACL and its secondary restraint. Knee Surg Sports Traumatol Arthrosc. 2012;20(5):870-7.

24. Parson EM, Gee AO, Spiekerman C, Cavanagh PR. The biomechanical function of the anterolateral ligament of the knee. Am J Sports Med. 2015;43(8):669-74.

25. Tanaka M, Vyas D, Moloney G, Bedy A, Pearle AD, Musahl $\checkmark$. What does it take to have a high-grade pivot shift? Knee Surg Sports Traumatol Arthrosc. 2012;20(4):737-42. 
26. Song GY, Zhang $H$, Wu G, Zhang J, Liu X, Xue Z, et al. Patients with high-grade pivot-shift phenomenon are associated with higher prevalence of anterolateral ligament injury after acute anterior cruciate ligament injuries. Knee Surg Sports Traumatol Arthrosc. 2017 Apr;25(4):1111-6.

27. Claes S, Luyckx T, Vereecke E, Bellemans J. The Segond fracture: a bony injury of the anterolateral ligament of the knee. Arthroscopy. 2014;30(11):1475-82.

28. Porrino J Jr, Maloney E, Richardson M, Mulcahy $\mathrm{H}$, $\mathrm{Ha}$ A, Chew FS. The anterolateral ligament of the knee: MRI appearance, association with the Segond fracture, and historical perspective. AJR Am J Roentgenol. 2015;204(2):367-73.

29. Helito CP, Helito PV, Costa HP, Bordalo-Rodrigues M, Pecora JR, Camanho GL, Demange MK. MRI evaluation of the anterolateral ligament of the knee: assessment in routine 1.5-T scans. Skeletal Radiol. 2014:43:1421-7.

30. Kosy JD, Mandalia VI, Anaspure R. Characterization of the anatomy of the anterolateral ligament of the knee using magnetic resonance imaging. Skeletal Radiol. 2015;44:1647-53.

31. Cavaignac E, Wytrykowski K, Reina N, Pailhé R, Murgier J, Faruch M, Chiron P. Ultrasonographic Identification of the Anterolateral Ligament of the Knee. Arthroscopy. 2016;32:120-6.

32. Oshima T, Nakase J, Numata H, Takata $Y$, Tsuchiya $H$. Ultrasonography imaging of the anterolateral ligament using real-time virtual sonography. Knee. 2016;23: 198-202.

33. Capo J, Kaplan DJ, Fralinger DJ, Adler RS, Campbell KA, Jazrawi LM, Alaia MJ. Ultrasonographic visualization and assessment of the anterolateral ligament. Knee Surg Sports Traumatol Arthrosc. 2017 Oct;25(10):3134-9.

34. Galway RD, Beaupre A, Maclntosh DL. Pivot shift: a clinical sign of symptomatic anterior cruciate insufficiency. J Bone Joint Surg Br. 1972;54B:763-4.

35. Sonnery-Cottet B, Daggett M, Helito CP, Fayard JM, Thaunat M. Combined Anterior Cruciate Ligament and Anterolateral Ligament Reconstruction. Arthrosc Tech. 2016 Oct 31;5(6).

36. Chahla J, Menge TJ, Mitchell JJ, Dean CS, LaPrade RF. Anterolateral ligament reconstruction technique: an anatomic-based approach. Arthrosc Tech. 2016;5(3): e453-457.

37. Wagih AM, Elguindy AM. Percutaneous Reconstruction of the Anterolateral Ligament of the Knee With a Polyester Tape. Arthrosc Tech. 2016;5(4):e691-e697.

38. Sonnery-Cottet B, Thaunat M, Freychet B, Pupim BH, Murphy CG, Claes S. Outcome of a combined anterior cruciate ligament and anterolateral ligament recon- struction technique with a minimum 2-year follow-up. Am J Sports Med. 2015;43:1598-605.

39. Kosy J, Soni A, Venkatesh R, Mandalia V. The anterolateral ligament of the knee: unwrapping the enigma. Anatomical study and comparison to previous reports. J Orthop Traumatol. 2016;17(4):303-8.

40. Helito CP, Miyahara HS, Bonadio MB, et al. Anatomical study of the anterolateral knee ligament. Revista Brasileira de Ortopedia. 2013;48:368-73.

41. Corbo G, Norris M, Getgood A, Burkhart T. The infra-meniscal fibers of the anterolateral ligament are stronger and stiffer tan the supra-meniscal fibers despite similar histological characteristics. Knee Surg Sports Traumatol Arthrosc. 2017;25(4):1078-85.

42. Feucht MJ, Bigdon S, Bode G, Salzmann GM, Dovi-Akue D, Südkamp NP, Niemeyer P. Associated tears of the lateral meniscus in anterior cruciate ligament injuries: risk factors for di erent tear patterns. J Orthop Surg Res. 2015;10:1-8.

43. Kilcoyne KG, Dickens JF, Haniuk E, Cameron KL, Owens $\mathrm{BD}$. Epidemiology of meniscal injury associated with $\mathrm{ACL}$ tears in young athletes. Orthopedics. 2012;35(3):208-12.

44. Van Dyck P, Clockaerts S, Vanhoenacker FM, Lambrecht V, Wouters K, de Smet E, et al. Anterolateral ligament abnormalities in patients with acute anterior cruciate ligament rupture are associated with lateral meniscal and osseous injuries. Eur Radiol. 2016;26(10):1-9.

45. Daggett M, Helito C, Cullen M, Ockuly A, Busch K, Granite J, et al. The anterolateral ligament: an anatomic study on sex-based differences. Orthop J Sports Med. 2017;5(2):2325967116689387.

46. Kittl C, Halewood C, Stephen JM, Gupte CM, Weiler A, Williams A, Amis AA. Length change patterns in the lateral extra-articular structures of the knee and related reconstructions. Am J Sports Med. 2015;43(2):354-62.

47. Imbert P, Lutz C, Daggett M, Niglis L, Freychet B, Dalmay F, Sonnery-Cottet B. Isometric characteristics of the anterolateral ligament of the knee: a cadaveric navigation study. Arthroscopy. 2016:32(10):2017-24.

48. Helito CP, Helito PV, Bonadio MB, da Mota E Albuquerque RF, Bordalo-Rodrigues M, Pecora JR, et al. Evaluation of the length and isometric pattern of the anterolateral ligament with serial computer tomography. Orthop J Sports Med. 2014;2:2325967114562205.

49. Sonnery-Cottet B, Lutz C, Daggett M, Dalmay F, Freychet $B$, Niglis L, Imbert P. The involvement of the anterolateral ligament in rotational control of the knee. Am J Sports Med. 2016;44(5):1209-14.

50. Kittl C, El-Daou H, Athwal KK, Gupte CM, Weiler A, Williams A, Amis AA. The role of the anterolateral structures and the $A C L$ in controlling laxity of the intact and 
ACL-deficient knee: response. Am J Sports Med. 2016 Apr;44(4):NP15-8.

51. Saiegh YA, Suero EM, Guenther D, Hawi N, Decker S, Krettek $C$, et al. Sectioning the anterolateral ligament did not increase tibiofemoral traslation or rotation in an ACL-deficient cadaveric model. Knee Surg Sport Traumatol Arthrosc. 2017;25(4):1086-92.

52. Thein R, Boorman-Padgett J, Stone K, Wickiewicz TL, Imhauser CW, Pearle AD. Biomechanical assessment of the anterolateral ligament of the knee a secondary restraint in simulated test of the pivot-shift and of anterior stability. J Bone Joint Surg Am. 2016;98(11):937-43.

53 Rahnemai-Azar AA, Miller RM, Guenther D, Fu FH, Lesniak BP, Musahl V, Debski RE. Structural properties of the anterolateral capsule and iliotibial band of the knee. Am J Sports Med. 2016;44(4):892-7.

54. Bonanzinga T, Signorelli C, Grassi A, Lopomo N, Bragonzoni L, Zaffagnini S, Marcacci M. Kinematics of ACL and anterolateral ligament. Part I: Combined lesion. Knee Surg Sports Traumatol Arthrosc. 2017;25(4):1055-61.

55. Yamamoto Y, Hsu WH, Fisk JA, Van Scyoc AH, Miura K, Woo SL. Effect of the iliotibial band on knee biomechanics during a simulated pivot shift test. J Orthop Res. 2006;24(5):967-73.

56. Hassler H, Jakob RP. Ein Beitrag zur Ursache der anterolateralen Instabilität des Kniegelenkes. Arch Orthop Trauma Surg. 1981;98(1):45-50.

57. Jakob RP, Noesberger B, Saxer U. [Value of the pivot-shift phenomenon and lateral reconstruction; diagnosis and therapy of anterior cruciate ligament rupture]. Schweiz Z Sportmed. 1977;25(2):69-84.

58. Lane JG, Irby SE, Kaufman K, Rangger C, Daniel DM. The anterior cruciate ligament in controlling axial rotation. An evaluation of this effect. Am J Sports Med. 1994;22(2):289-93.

59. Wroble RR, Grood ES, Cummings JS, Henderson JM, Noyes FR. The role of the lateral extraarticular restraints in the anterior cruciate ligament-deficient knee. Am J Sports Med. 1993;21(2):257-62.

60. Musahl V, Citak M, O'Loughlin PF, Choi D, Bedi A, Pearle $A D$. The effects of medial versus lateral meniscectomy on the stability of the anterior cruciate ligament knee. Am J Sports Med. 2010;38(8):1591-7.

61. Diermann N, Schumacher T, Schanz S, Rascheke MJ, Petersen W, Zantop T. Rotational instability of the knee: internal tibial rotation under a simulated pivot-shift test. Arch Orthop Trauma Surg. 2009;129(3):353-8.

62. Song GY, Zhang H, Wang QQ, Zhang J, Li Y, Feng H. Bone Contusions After Acute Noncontact Anterior Cruciate Ligament Injury Are Associated With Knee Joint Laxity, Concomitant Meniscal Lesions, and Anterolateral
Ligament Abnormality. Arthroscopy. 2016 Nov;32(11): 2331-41.

63. Song GY, Zhang H, Wang QQ, Zhang J, Li Y, Feng H. Risk Factors Associated With Grade 3 Pivot Shift After Acute Anterior Cruciate Ligament Injuries. Am J Sports Med. 2016 Feb;44(2):362-9.

64. Musahl V, Rahnemai-Azar AA, Costello J, Arner JW, Fu $\mathrm{FH}$, Hoshino Y, et al. The Influence of Meniscal and Anterolateral Capsular Injury on Knee Laxity in Patients With Anterior Cruciate Ligament Injuries. Am J Sports Med. 2016 Dec;44(12):3126-31.

65. Huser LE, Noyes FR, Jurgensmeier D, Levy MS. Anterolateral Ligament and Iliotibial Band Control of Rotational Stability in the Anterior Cruciate Ligament-Intact Knee: Defined by Tibiofemoral Compartment Translations and Rotations. Arthroscopy. 2017 Mar;33(3):595-604.

66. Ferretti A, Monaco E, Fabbri M, Maestri B, De Carli A. Prevalence and Classification of Injuries of Anterolateral Complex in Acute Anterior Cruciate Ligament Tears. Arthroscopy. 2017 Jan;33(1):147-54.

67. Woods GW, Stanley RF, Tullos HS. Lateral capsular sign: $X$-ray clue to a significant knee instability. Am J Sports Med. 1979;7(1):27-33.

68. Goldman AB, Pavlov H, Rubenstein D. The Segond fracture of the proximal tibia: a small avulsion that reflects major ligamentous damage. AJR Am J Roentgenol. 1988;151(6):1163-67.

69. Hess T, Rupp S, Hopf T, Gleitz M, Liebler J. Lateral tibial avulsion fractures and disruptions to the anterior cruciate ligament. A clinical study of their incidence and correlation. Clin Orthop Relat Res. 1994;(303):193-97.

70. Arneja SS, Furey MJ, Alvarez CM, Reilly CW. Segond fractures: not necessarily pathognomonic of anterior cruciate ligament injury in the pediatric population. Sports Health. 2010;2(5):437-9.

71. Irvine GB, Dias JJ, Finlay DB. Segond fractures of the lateral tibial condyle: Brief report. J Bone Joint Surg Br. 1987;69(4):613-4.

72. De Maeseneer M, Boulet C, Willekens I, Lenchik L, De Mey J, Cattrysse E, Shahabpour M. Segond fracture: Involvement of the iliotibial band, anterolateral ligament, and anterior arm of the biceps femoris in knee trauma. Skeletal Radiol. 2015;44:413-21.

73. Klos B, Scholtes M, Konijnenberg S. High prevalence of all complex Segond avulsion using ultrasound imaging. Knee Surg Sports Traumatol Arthrosc. 2017 Apr;25(4):1331-8.

74. Helito CP, Demange MK, Helito PV, Costa HP, Bonadio $M B$, Pecora JR, et al. Evaluation of the anterolateral ligament of the knee by means of magnetic resonance examination. Rev Bras Ortop. 2015 Apr 7;50(2):214-9. 
75. Taneja AK, Miranda FC, Braga CA, Gill CM, Hartmann LG, Santos DC, Rosemberg LA. MRI features of the anterolateral ligament of the knee. Skeletal Radiol. 2015;44:403-10.

76. Claes S, Bartholomeeusen S, Bellemans J. High prevalence of anterolateral ligament abnormalities in magnetic resonance images of anterior cruciate ligament-injured knees. Acta Orthop Belg. 2014;80:45-9.

77. Helito CP, Helito PVP, Leão RV, Demange MK, Bordalo-Rodrigues M. Anterolateral ligament abnormalities are associated with peripheral ligament and osseous injuries in acute ruptures of the anterior cruciate ligament. Knee Surg Sports Traumatol Arthrosc. 2017 Apr;25(4):1140-8.

78. Helito CP, Helito PVP, Bonadio MB, Pécora JR, Bordalo-Rodrigues M, Camanho GL, Demange MK. Correlation of magnetic resonance imaging with knee anterolateral ligament anatomy: a cadaveric study. Orthop J Sports Med. 2015 Dec 16;3(12):2325967115621024.

79. Hartigan DE, Carroll KW, Kosarek FJ, Piasecki DP, Fleischli JF, D'Alessandro DF. Visibility of Anterolateral Ligament Tears in Anterior Cruciate Ligament-Deficient Knees With Standard 1.5-Tesla Magnetic Resonance Imaging. Arthroscopy. 2016 Oct;32(10):2061-5.

80. Devitt BM, O'Sullivan R, Feller JA, Lash N, Porter TJ, Webster KE, Whitehead TS. MRI is not reliable in diagnosing of concomitant anterolateral ligament and anterior cruciate ligament injuries of the knee. Knee Surg Sports Traumatol Arthrosc. 2017 Apr;25(4):1345-51.

81. Lemaire M, Combelles F. Plastic repair with fascia lata for old tears of the anterior cruciate ligament. Rev Chir Orthop Reparatrice Appar Mot. 1980 NovDec;66(8):523-5.

82. Losee RE, Johnson TR, Southwick WO. Anterior subluxation of the lateral tibial plateau. A diagnostic test and operative repair. J Bone Joint Surg Am. 1978;60(8): 1015-30.

83. Ellison AE. Distal iliotibial-band transfer for anterolateral rotatory instability of the knee. J Bone Joint Surg Am. 1979;61(3):330-7.

84. Andrews JR, Sanders R. A “mini-reconstruction" technique in treating anterolateral rotatory instability (ALRI). Clin Orthop Relat Res. 1983;(172):93-6.

85. Duthon VB, Magnussen RA, Servien E, Neyret P. ACL reconstruction and extra-articular tenodesis. Clin Sports Med. 2013 Jan;32(1):141-53.

86. Strum GM, Fox JM, Ferkel RD, Dorey FH, Del Pizzo W, Friedman MJ, et al. Intraarticular versus intraarticular and extraarticular reconstruction for chronic anterior cruciate ligament instability. Clin Orthop Relat Res. 1989 Aug;(245):188-98.
87. Ménétrey J, Duthon VB, Laumonier T, Fritschy D. “Biological failure" of the anterior cruciate ligament graft. Knee Surg Sports Traumatol Arthrosc. 2008 Mar;16(3):224-31.

88. Yagi M, Wong EK, Kanamori A, Debski RE, Fu FH, Woo SL. Biomechanical analysis of an anatomic anterior cruciate ligament reconstruction. Am J Sports Med. 2002 Sep-Oct;30(5):660-6.

89. Caborn DN, Chang HC. Single femoral socket double-bundle anterior cruciate ligament reconstruction using tibialis anterior tendon: description of a new technique. Arthroscopy. 2005 Oct;21(10):1273.

90. Espejo-Baena A, Serrano-Fernández JM, de la TorreSolís F, Irizar-Jiménez S. Anatomic double-bundle ACL reconstruction with femoral cortical bone bridge support using hamstrings. Knee Surg Sports Traumatol Arthrosc. 2009 Feb;17(2):157-61.

91. Colombet PD, Robinson JR. Computer-assisted, anatomic, double-bundle anterior cruciate ligament reconstruction. Arthroscopy. 2008 Oct;24(10):1152-60.

92. Zaffagnini S, Marcheggiani Muccioli GM, Lopomo N, Signorelli C, Bonanzinga T, Musiani C, et al. Can the pivot-shift be eliminated by anatomic double-bundle anterior cruciate ligament reconstruction? Knee Surg Sports Traumatol Arthrosc. 2012;20(4):743-51.

93. Branch TP, Siebold R, Freedberg HI, Jacobs CA. Double-bundle $A C L$ reconstruction demonstrated superior clinical stability to single-bundle ACL reconstruction: a matched-pairs analysis of instrumented tests of tibial anterior translation and internal rotation laxity. Knee Surg Sports Traumatol Arthrosc. 2011 Mar;19(3).

94. Mascarenhas R, Cvetanovich GL, Sayegh ET, Verma NN, Cole BJ, Bush-Joseph C, Bach BR Jr. Does Double-Bundle Anterior Cruciate Ligament Reconstruction Improve Postoperative Knee Stability Compared With Single-Bundle Techniques? A Systematic Review of Overlapping Meta-analyses. Arthroscopy. 2015 Jun;31(6):1185-96.

95. Järvelä S, Kiekara T, Suomalainen, Järvelä T. Double-Bundle Versus Single-Bundle Anterior Cruciate Ligament Reconstruction: A Prospective Randomized Study With 10-Year Results. Am J Sports Med. 2017 Sep;45(11):2578-85.

96. Espejo-Baena A, Espejo-Reina A. Anatomic outside-in anterior cruciate ligament reconstruction using a suspension device for femoral fixation. Arthrosc Tech. 2014 Apr 13;3(2):e265-9.

97. Colombet P, Graveleau N. An Anterior Cruciate Ligament Reconstruction Technique With 4-Strand Semitendinosus Grafts, Using Outside-In Tibial Tunnel Drilling and Suspensory Fixation Devices. Arthrosc Tech. 2015 Sep 28;4(5):e507-11. 
98. Leiter JR, Gourlay R, McRae S, de Korompay N, MacDonald PB. Long-term follow-up of ACL reconstruction with hamstring autograft. Knee Surg Sports Traumatol Arthrosc. 2014 May;22(5):1061-9.

99. Crawford SN, Waterman BR, Lubowitz JH. Long-term failure of anterior cruciate ligament reconstruction. Arthroscopy. 2013;29:1566-71.

100. Kocher MS, Steadman JR, Briggs K, Zurakowski D, Sterett WI, Hawkins RJ. Determinants of patient satisfaction with outcome after anterior cruciate ligament reconstruction. J Bone Joint Surg. 2002;84-A(9):1560-72.

101. Katakura M, Koga H, Nakamura K, Sekiya I, Muneta T. Effects of different femoral tunnel positions on tension changes in anterolateral ligament reconstruction. Knee Surg Sports Traumatol Arthrosc. 2017 Apr;25(4):1272-8.

102. Helito CP, Bonadio MB, Rozas JS, Wey JM, Pereira CA, Cardoso TP, et al. Biomechanical study of strength and stiffness of the knee anterolateral ligament. BMC Musculoskelet Disord. 2016;17:193.

103. Monaco E, Lanzetti RM, Fabbri M, Redler A, De Carli A, Ferretti A. Anterolateral ligament reconstruction with autologous grafting: a biomechanical study. Clin Biomech (Bristol, Avon). 2017 May;44:99-103.

104. Vadalà AP, Iorio R, De Carli A, Bonifazi A, Iorio C, Gatti A, et al. An extra-articular procedure improves the clinical outcome in anterior cruciate ligament reconstruction with hamstrings in female athletes. Int Orthop. 2013;37:187-92.

105. Dejour D, Vanconcelos W, Bonin N, Saggin PR. Comparative study between mono-bundle bone-patellar tendon-bone, double-bundle hamstring and monobundle bone-patellar tendon-bone combined with a modified Lemaire extra-articular procedure in anterior cruciate ligament reconstruction. Int Orthop. 2013;37:193-9.

106. Trojani C, Beaufils P, Burdin G, Bussière C, Chassaing $V$, Djian $\mathrm{P}$, et al. Revision $\mathrm{ACL}$ reconstruction: influence of a lateral tenodesis. Knee Surg Sports Traumatol Arthrosc. 2012;20:1565-70.

107. Monaco E, Labianca L, Conteduca F, De Carli A, Ferretti A. Double bundle or single bundle plus extra-articular tenodesis in ACL reconstruction? A CAOS study. Knee Surg Sports Traumatol Arthrosc. 2007;15:1168-74.

108. Anderson AF, Snyder RB, Lipscomb AB. Anterior cruciate ligament reconstruction. A prospective randomized study of three surgical methods. Am J Sports Med. 2001;29:272-9.

109. Roth JH, Kennedy JC, Lockstadt H, McCallum CL, Cunning LA. Intra-articular reconstruction of the anterior cruciate ligament with and without extra-articular supplementation by transfer of the biceps femoris tendon. J Bone Joint Surg. 1987;69:275-8.

110. Stentz-Olesen K, Nielsen ET, de Raedt S, Jørgensen $\mathrm{PB}, \mathrm{S} \phi$ rensen OG, Kaptein B, et al. Reconstructing the anterolateral ligament does not decrease rotational knee laxity in ACL-reconstructed knees. Knee Surg Sports Traumatol Arthrosc. 2017 Apr;25(4):1125-31.

111. Nielsen S, Ovesen J, Rasmussen O. The anterior cruciate ligament of the knee: an experimental study of its importance in rotatory knee instability. Arch Orthop Trauma Surg. 1984;103(3):170-4.

112. Lane JG, Irby SE, Kaufman K, Rangger C, Daniel DM. The anterior cruciate ligament in controlling axial rotation. An evaluation of its effect. Am J Sports Med. 1994;22(2):289-93.

113. O'Neill DB. Arthroscopically assisted reconstruction of the anterior cruciate ligament. A prospective randomized analysis of three techniques. J Bone Joint Surg Am. 1996;78:803-13.

114. Piasecki DP, Bach BR Jr, Espinoza Orias AA, Verma NN. Anterior cruciate ligament reconstruction: can anatomic femoral placement be achieved with a transtibial technique? Am J Sports Med. 2011;39:1306-15.

115. Loh JC, Fukuda Y, Tsuda E, Steadman RJ, Fu FH, Woo SL. Knee stability and graft function following anterior cruciate ligament reconstruction: comparison between 11 o'clock and 10 o'clock femoral tunnel placement. 2002 Richard O'Connor Award paper. Arthroscopy. 2003;19:297-304.

116. Siebold R. The concept of complete footprint restoration with guidelines for single- and double-bundle $\mathrm{ACL}$ reconstruction. Knee Surg Sports Traumatol Arthrosc. 2011;19:699-706.

117. Śmigielski R, Zdanowicz $U$, Drwięga M, Ciszek B, Williams $A$. The anatomy of the anterior cruciate ligament and its relevance to the technique of reconstruction. Bone Joint J. 2016 Aug;98-B(8):1020-6.

118. Mochizuki T, Fujishiro H, Nimura A, Mahakkanukrauh $P$, Yasuda K, Muneta T, Akita K. Anatomic and histologic analysis of the mid-substance and fan-like extension fibres of the anterior cruciate ligament during knee motion, with special reference to the femoral attachment. Knee Surg Sports Traumatol Arthrosc. 2014 Feb;22(2):336-44.

119. US National Library of Medicine (US). Multicenter randomized clinical trial comparing anterior cruciate ligament reconstruction with and without lateral extraarticular tenodesis in individuals who are at high risk of graft failure. Bethesda, MD, December 2, 2013. Disponible en: https://clinicaltrials.gov/show/ NCT02018354. 